\title{
Single Parameter Adaptive Control of Unknown Nonlinear Systems with Tracking Error Constraints
}

\author{
Hongjun Yang $\mathbb{D},{ }^{1}$ Zhijie Liu $\mathbb{D},^{2}$ and Shuang Zhang $\mathbb{D}^{3}$ \\ ${ }^{1}$ State Key Laboratory of Management and Control for Complex System, Institute of Automation, Chinese Academy of Sciences, \\ Beijing 100190, China \\ ${ }^{2}$ School of Automation Science and Electrical Engineering, Beihang University, Beijing 100191, China \\ ${ }^{3}$ School of Automation and Electrical Engineering, University of Science and Technology Beijing, Beijing 100083, China
}

Correspondence should be addressed to Zhijie Liu; liuzhijie2012@gmail.com and Shuang Zhang; zhangshuang.ac@gmail.com Received 23 March 2018; Accepted 25 July 2018; Published 2 October 2018

Academic Editor: Andy Annamalai

Copyright (C) 2018 Hongjun Yang et al. This is an open access article distributed under the Creative Commons Attribution License, which permits unrestricted use, distribution, and reproduction in any medium, provided the original work is properly cited.

\begin{abstract}
This paper investigates a single parameter adaptive neural network control method for unknown nonlinear systems with bounded external disturbances. A smooth performance function is developed to achieve the transient and steady state of system tracking error that could be constrained in prescribed bounds. The difficulties in dealing with unknown system parameters and disturbances of nonlinear systems are resolved based on the single parameter adaptive neural network control which is proposed to effectively reduce the calculation amount. The theoretical analysis implies that the proposed control scheme makes the closed-loop system uniformly ultimately bounded. Simulation demonstrates that the proposed adaptive controller gives a favorable performance on tracking desired signal and constraining the bounds of tracking error which could be arbitrarily small with appropriate adaptive parameters. Both the theoretical analysis and simulations confirm the effectiveness of the control scheme.
\end{abstract}

\section{Introduction}

Neural networks (NNs) have been widely applied to estimate continuous unknown functions in complex and modeluncertain systems [1-5]. The primary advantage of $\mathrm{NN}$ techniques is that the controller does not require the accurate system information. During the recent researches, adaptive control was commonly used to combine with neural control [6-10], in particular with $\mathrm{RBF}$ NNs [11-13]. A direct adaptive controller [11] based on an improved RBF NN was proposed for an omnidirectional mobile robot. In [12], a model reference adaptive sliding mode using an RBF NN was proposed to control the single-phase active power filter. An adaptive fault-tolerant attitude control method [13] was presented of rigid body using RBF NN.

Although the stabilities of system are guaranteed by using adaptive RBF NN control design techniques and tracking errors of output could converge in the finite time, a desired performance of transient and steady state cannot be easily obtained. However, the transient and steady state performance plays a significant role when some control requirements are raised such as avoiding large overshoot. These requirements stimulate tracking-error-constrained developments such as the barrier Lyapunov function method [14-17], adaptive inverse of backlash [18], and the error transformation method [19-22]. In order to obtain the prescribed transient and steady state performance, we use a smooth function to regulate constraints in this study.

Recently, we have made some achievements on adaptive NN control $[23,24]$ and constrained control $[25,26]$. In this study, we consider an adaptive NN control for unknown nonlinear systems with tracking error constraints. Our control objective is to develop the controller achieving the desired trajectory tracking and constraining the tracking error acting as our required performance. First, we use a smooth function which could regulate constraints such as the convergence speed, the maximum bounds of overshoot, and transient and steady state of the tracking error. Then, the constrained function is transformed to an unconstrained variable to facilitate the controller design. To proceed, an 
RBF NN, combined with the single parameter adaptive (SPA) control method, is designed to address the functionunknown issue of the system. The main contributions include the following three aspects:

(1) The system tracking error could be constrained in the required bounds. The transient and steady state, convergence speed, and maximum bound of overshoot of the tracking error could be arbitrarily prescribed and regulated by the parameters of the proposed smooth performance function

(2) Generally, NN weight vectors were used as the adaptive parameters in many literatures [27, 28]. To improve it and decrease the online computation, we design a single parameter adaptive (SPA) control method. We use only one parameter to estimate the norm of weight vector online, shortening the calculated time. Meanwhile, we do not have to consider the structure of weight vector but a single parameter instead, which also simplifies the design of the controller.

(3) The research object is a nonlinear system, with unknown functions as well as external disturbances, which increases difficulties in designing the effective controller. Whereas the proposed controller is concise and the closed-loop system is guaranteed uniformly ultimately bounded

A coupled motor drive (CMD) system [29], generally applied in intelligent robots [30], is taken for simulation, which displays the favorable tracking performance of output under the designed controller. The effectiveness of the developed scheme is validated, and the convergence rate of the tracking error could be arbitrarily small through selecting the appropriate parameters.

The paper is organized as follows. Section 2 gives the model structure of the SISO nonlinear system, and then the performance function is also proposed. Section 3 presents an ideal controller. A single parameter adaptive neural network control method is introduced in Section 4, where the stability of the closed-loop system and the favorable performance of the control scheme are all guaranteed. Numerical results are shown in Section 5 to illustrate the effectiveness of the control scheme. Finally, the conclusion is presented in Section 6.

\section{System Description and Preliminaries}

Consider a class of unknown single-input single-output unknown nonlinear system as follows:

$$
\begin{aligned}
& \dot{x}_{i}=x_{i+1}, \quad i=1,2, \ldots, n-1, \\
& \dot{x}_{n}=f(x)+b(x) u+d(t), \\
& y=x_{1},
\end{aligned}
$$

where $x=\left[x_{1}, x_{2}, \ldots, x_{n}\right]^{\mathrm{T}} \in R^{n}, u \in R$, and $y \in R$ are, respectively, the state, input, and output of the system; $f(x)$ and $b(x)$ denote unknown nonlinear smooth functions; and $d(t)$ represents the unknown disturbance bounded as $|d(t)| \leq d_{1}$, where $d_{1}$ is a positive constant. As all values of actual physical quantities in (1) are limited, $x$ lies in a subset $\Phi \in R^{\mathrm{n}}$.

The control goal is designing the control input $u$ such that the output $y$ could track the desired trajectory $y_{d}$ and the tracking error $e(t)=y-y_{d}$ acts as our required performance. For the second purpose, we first define a smooth function as

$$
\mu(t)=\left(\mu_{0}-\mu_{\infty}\right) \exp (-l t)+\mu_{\infty}
$$

where $\mu_{0}, \mu_{\infty}$, and $l$ are positive constants and $\mu_{\infty}<\mu_{0}$. Meanwhile, an asymmetric hyperbolic tangent function $T(\xi)$ is defined as follows:

$$
T(\xi)= \begin{cases}\frac{\exp (\xi)-\sigma \exp (-\xi)}{\exp (\xi)+\exp (-\xi)}, & \text { if } e(0) \geq 0 \\ \frac{\sigma \exp (\xi)-\exp (-\xi)}{\exp (\xi)+\exp (-\xi)}, & \text { if } e(0)<0\end{cases}
$$

where $0 \leq \sigma \leq 1$ is a design constant and $\xi$ is the transformed error variable. Obviously, $T(\xi)$ possesses the following properties:

$$
\begin{cases}-\sigma<T(\xi)<1, & \text { if } e(0)>0 \\ -1<T(\xi)<\sigma, & \text { if } e(0)<0\end{cases}
$$

We define the following equation:

$$
e(t)=\mu(t) T(\xi)
$$

From (2), (3), and (5), we conclude that when $e(0) \geq 0$, it yields

$$
-\sigma \mu(t)<e(t)<\mu(t)
$$

and when $e(0)<0$, we obtain

$$
-\mu(t)<e(t)<\sigma \mu(t)
$$

Remark 1. The smooth function $\mu(t)$ defined in (2) is designed as the guidance of the tracking error $e$ such that the performance of $e$ is constrained and complies with the performance of $\mu(t)$. To be specific, the constant $\mu_{\infty}$ denotes the upper bound of $|e|$ at the steady state, and the constant $l$ represents the decreasing rate of $\mu(t)$, i.e., the bound of prescribed convergence speed of $e$, assuming that $|e(0)|<\mu_{0}$. In addition, $\sigma \mu(0)$ is the bound of the required maximum overshoot.

Remark 2. $T(\xi)$ is an error transforming function for obtaining an unconstrained tracking error $\xi$ from the constrained system (5), and $T(\xi)$ is a smooth and increasing function. 
As the properties of $T(\xi)$, we could get the inverse function of $T(\xi)$ as follows:

$$
\xi=T^{-1}\left(\frac{e(t)}{\mu(t)}\right)
$$

where $\xi \in L_{\infty}$ and the tracking error $e$ is constrained in the set:

$$
\mathrm{E}=\left\{e \in R:|e(t)|<\mu_{\infty}\right\} .
$$

Thus, if we guarantee $\xi$ bounded $\forall t \geq 0$, we can realize the fact that $y$ tracks the desired trajectory $y_{d}$ and the tracking error $e$ acts as our prescribed performance. In addition, the required performance of the system output is regulated by the appropriate choice of $\mu(t)$ and $\sigma$.

Define the filtered error function $s$ as

$$
s=c_{1} \xi+c_{2} \dot{\xi}+\cdots+c_{n-1} \xi^{(n-2)}+\xi^{(n-1)},
$$

where $c_{i}, i=1,2, \ldots, n-1$, is selected as $c_{i}>0$ such that the polynomial $\lambda^{n-1}+c_{n-1} \lambda^{n-2}+\cdots+c_{2} \lambda+c_{1}$ is Hurwitz. Then, we could confirm that $\xi$ is bounded if we guarantee the convergence of the filtered error function $s$ [31]. Thus, the transient and steady state, convergence speed, and maximum bound of overshoot of the tracking error could be arbitrarily prescribed constrained and regulated by the parameters of the proposed smooth performance function.

Assumption 1. The known desired trajectory signal $y_{d} \in$ $\Phi_{d} \subset R^{n}$ and its first to $n$th order time derivatives are continuous and bounded, where $\Phi_{d}$ is a connected subset of $\Phi$.

From (1), (5), and (7), differentiating $s$ with respect to time, we have

$$
\begin{aligned}
\dot{s} & =c_{1} \dot{\xi}+c_{2} \ddot{\xi}+\cdots+c_{n-1} \xi^{(n-1)}+\xi^{(n)} \\
& =v+\frac{\partial T^{-1}}{\partial(e(t) / \mu(t))} \frac{1}{\mu(t)} \dot{x}_{n} \\
& =v+w+F u+d_{f}(t),
\end{aligned}
$$

where $v$ is the term containing the following known variables: $e, \dot{e}, \ldots, e^{(n-1)}, \mu, \dot{\mu}, \ldots, \mu^{(n)}, y_{d}^{(n)}$, and the unknown term $w$ $=\left(\partial T^{-1} /(\partial(e(t) / \mu(t)))\right)(1 /(\mu(t))) f(x), \quad F=\left(\partial T^{-1} /(\partial(e(t) /\right.$ $\mu(t))))(1 /(\mu(t)))(1 /(\mu(t)))(b(x)), \quad d_{f}(t)=\left(\partial T^{-1} /(\partial(e(t) /\right.$ $\mu(t))))(1 /(\mu(t)))(d(t))$.

Assumption 2. The sign of $b(x)$ is knowable and $|b(x)|>0$, $\forall x \in \Phi$. It is assumed that $b(x)>0$. As the fact of $\left(\partial T^{-1} /\right.$ $(\partial(e(t) / \mu(t))))(1 /(\mu(t)))>0$, we have $F>0$.

Assumption 3. It could be found a smooth function $\bar{F}$ such that $|F| \leq \bar{F}, \bar{F} \leq \bar{F}_{b} \in R$. The disturbance $d_{f}(t)$ is bounded by a known positive constant $d_{0}$, i.e., $\left|d_{f}(t)\right| \leq d_{0}$.

Remark 3. The restrictions of Assumptions 2 and 3 are actually the same as the ones in the literature [27]. Many robotic systems, including CMD system in this paper, possess the properties as those of Assumptions 2 and 3.

\section{Design of Ideal Controller}

To design ideal controller $\widehat{u}$, we assume that the unknown functions $f(x)$ and $b(x)$ are known and $d(t)=0$.

Theorem 1. Given the system (1), Assumptions 1-3, the ideal controller is developed by

$$
\widehat{u}=-\frac{1}{F}\left\{v+w+\left[\frac{1}{\delta}+\frac{1}{\delta F}-\frac{\dot{F}}{2 F}\right] s\right\},
$$

where $\delta$ is a positive parameter. Then, we have $\lim _{t \rightarrow \infty}|s|=0$.

Proof 1. Substituting $u=\widehat{u}$ into (11), we get

$$
\dot{s}=-\left[\frac{1}{\delta}+\frac{1}{\delta F}-\frac{\dot{F}}{2 F}\right] s .
$$

Define a Lyapunov candidate as $V^{\prime}=(1 / 2 F) s^{2}$, then differentiating it with respect to time yields

$$
\begin{aligned}
\dot{V}^{\prime} & =\frac{1}{F} \dot{s} \dot{\mathcal{S}}-\frac{\dot{F}}{2 F^{2}} s^{2} \\
& =-\frac{1}{F}\left[\frac{1}{\delta}+\frac{1}{\delta F}-\frac{\dot{F}}{2 F}\right] s^{2}-\frac{\dot{F}}{2 F^{2}} s^{2} \\
& =-\left(\frac{1}{\delta F}+\frac{1}{\delta F^{2}}\right) s^{2} \leq 0 .
\end{aligned}
$$

According to the Lyapunov theorem, we obtain $\lim _{t \rightarrow \infty}|s|=0$.

From (14), we can conclude that the smaller $\delta$ is, the bigger convergence rate will be. Owning to the unknown functions $f(x)$ and $b(x), \widehat{u}$ is also unknown. Then, NN will be applied to develop the actual controller $u$ in the following section.

\section{Design of Actual Controller}

We rewrite the ideal controller (12) to the following form, in which the ideal controller $\widehat{u}$ is regarded as a function:

$$
\widehat{u}=u(\psi), \quad \psi=\left[x^{\mathrm{T}} v s\right]^{\mathrm{T}} \in \Phi_{\psi} \subset R^{n+2},
$$

where the subset $\Phi_{\psi}$ is expressed as

$$
\Phi_{\psi}=\left\{\left(\begin{array}{lll}
x^{\mathrm{T}} & v & s
\end{array}\right) \mid x \in \Phi, y_{\mathrm{d}} \in \Phi_{\mathrm{d}}\right\} .
$$

It is well known that continuous functions could be approximated by a linear combination of Gaussians. Because 
$\widehat{u}$ is continuous on $\Phi_{\psi}$ in (15), it has an ideal NN weight vector $\bar{W}$ as follows:

$$
\widehat{u}(\psi)=\bar{W}^{\mathrm{T}} h(\psi)+\omega_{\varepsilon}
$$

where $\omega_{\varepsilon}$ denotes the approximation error as $\left|\omega_{\varepsilon}\right| \leq \omega_{0}$, $\omega_{0}>0$, and $h(\psi)$ denotes the radial-basis function vector as

$$
\begin{aligned}
& h=\left[h_{1} h_{2} \cdots h_{j} \cdots h_{m}\right]^{\mathrm{T}}, \\
& h_{j}=\exp \left(-\frac{\left\|\psi-A_{j}\right\|^{2}}{b_{j}^{2}}\right), \quad j=1,2, \ldots, m,
\end{aligned}
$$

where $\psi \in R^{n+2}$ represents the input vector; $n+2$ denotes input neural nets number in the input layer; $m$ is hidden neural nets number in the hidden layer; $h_{j}$ is commonly used Gaussian function; $A_{j}=\left[a_{j}, a_{j}, \cdots, a_{j}\right]^{\mathrm{T}} \in R^{n+2}$; and $a \in R^{m}$ and $b_{j} \in R$, respectively, denote the vector of the center of the receptive field and the width of Gaussian function.

Assumption 4. There is a bound of ideal weight vector $\bar{W}$ such that

$$
\|\bar{W}\|_{F} \leq w_{\max }
$$

where $w_{\max }$ is a positive constant.

Then, a constant is introduced to improve the control scheme as

$$
\phi=\|\bar{W}\|_{F}^{2}
$$

Remark 4. As in Assumption 4, $\phi$ is also bounded.

Remark 5. $\phi$ is an unknown positive constant because of $\bar{W}$ being unknown.

We define $\widehat{\phi}$ as the estimate of $\phi$, then the estimate error is denoted as $\tilde{\phi}=\widehat{\phi}-\phi$. Then, the SPA control technique will be introduced to effectively reduce the calculation amount. We use only one parameter $\widehat{\phi}$ to estimate the norm of weight vector $\bar{W}$ online, instead of the estimate of a vector $\bar{W}$, which could considerably decrease the online computation. Meanwhile, we do not have to consider the structure of $\bar{W}$ but a single parameter $\widehat{\phi}$ instead, which also simplifies the design of the controller. It is one of the main contributions in our study.

First, we propose the controller as follows:

$$
u=-\frac{1}{2 \delta} s \widehat{\phi} h^{\mathrm{T}} h
$$

Substituting (21) into (11) yields

$$
\dot{s}=v+w+F\left(-\frac{1}{2 \delta} s \widehat{\phi} h^{\mathrm{T}} h\right)+d_{f}(t)
$$

From (17), we rewrite (22) by adding and subtracting $F \widehat{u}(\psi)$ simultaneously as

$$
\dot{s}=v+w+F\left(-\frac{1}{2 \delta} s \widehat{\phi} h^{\mathrm{T}} h-\bar{W}^{\mathrm{T}} h-\omega_{\varepsilon}\right)+F \widehat{u}(\psi)+d_{f}(t),
$$

and substituting (12) into (23), we get

$$
\dot{s}=F\left(-\frac{1}{2 \delta} s \widehat{\phi} h^{\mathrm{T}} h-\bar{W}^{\mathrm{T}} h-\omega_{\varepsilon}\right)-\left(\frac{1}{\delta}+\frac{1}{\delta F}-\frac{\dot{F}}{2 F}\right) s+d_{f}(t) .
$$

In order to validate all the states of system stabilizing in the compact subset $\Phi$, we propose the following theorem.

Theorem 2. On account of system (1) and Assumptions 1-4, with the SPA RBF NN controller (21), and the adaptive law:

$$
\dot{\hat{\phi}}=\frac{\gamma}{2 \delta} s^{2} h^{\mathrm{T}} h-\kappa \gamma \widehat{\phi}
$$

where $\gamma, \kappa>0$, the tracking error $e(t)$ is uniformly ultimately bounded in the compact subset $\Phi$ for $t \rightarrow \infty$ and can be arbitrarily small by using the appropriate parameter $\mu_{\infty}$. Moreover, the output $y=x_{1}$ in system (1) will track $y_{d}$ acting as our required performance.

Proof 2. A Lyapunov function candidate is defined as follows:

$$
V=\frac{1}{2}\left(\frac{s^{2}}{F}+\frac{1}{\gamma} \tilde{\phi}^{2}\right)
$$

Taking the time derivatives of (26), and from (24), we obtain

$$
\begin{aligned}
\dot{V}= & \frac{s \dot{s}}{F}-\frac{\dot{F}}{2 F^{2}} s^{2}+\frac{1}{\gamma} \tilde{\phi} \dot{\hat{\phi}} \\
= & \frac{s}{F}\left[F\left(-\frac{1}{2 \delta} s \widehat{\phi} h^{\mathrm{T}} h-\bar{W}^{\mathrm{T}} h-\omega_{\varepsilon}\right)\right] \\
& -\frac{\dot{F}}{2 F^{2}} s^{2}-\frac{s}{F}\left[\left(\frac{1}{\delta}+\frac{1}{\delta F}-\frac{\dot{F}}{2 F}\right) s-d_{f}(t)\right]+\frac{1}{\gamma} \tilde{\phi} \dot{\hat{\phi}} \\
= & -\frac{1}{2 \delta} s^{2}(\tilde{\phi}+\phi) h^{\mathrm{T}} h-s \bar{W}^{\mathrm{T}} h-\left(\frac{1}{\delta F}+\frac{1}{\delta F^{2}}\right) s^{2} \\
& +\frac{d_{f}(t)}{F} s-\omega_{\varepsilon} s+\frac{1}{\gamma} \tilde{\phi} \dot{\hat{\phi}}
\end{aligned}
$$


Noting the following inequalities:

$$
\begin{gathered}
\frac{s^{2} \phi h^{\mathrm{T}} h}{2 \delta}+\frac{\delta}{2}=\frac{s^{2}\|\bar{W}\|^{2} h^{\mathrm{T}} h}{2 \delta}+\frac{\delta}{2} \geq-s \bar{W}^{\mathrm{T}} h, \\
\left|\frac{d_{f}(t)}{F} s\right| \leq \frac{s^{2}}{\delta F^{2}}+\frac{\delta}{4}\left[d_{f}(t)\right]^{2}, \\
\left|\omega_{\varepsilon} s\right| \leq \frac{s^{2}}{2 \delta F}+\frac{\delta}{2} \omega_{\varepsilon}^{2} F,
\end{gathered}
$$

and from $\left|\omega_{\varepsilon}\right| \leq \omega_{0},\left|d_{f}(t)\right| \leq d_{0}$, and Assumption 3, we have

$$
\begin{aligned}
\dot{V} \leq & \tilde{\phi}\left(-\frac{1}{2 \delta} s^{2} h^{\mathrm{T}} h+\frac{1}{\gamma} \dot{\hat{\phi}}\right)-\left(\frac{1}{\delta F}+\frac{1}{\delta F^{2}}\right) s^{2} \\
& +\frac{d_{f}(t)}{F} s-\omega_{\varepsilon} s+\frac{\delta}{2} \\
\leq & \tilde{\phi}\left(-\frac{1}{2 \delta} s^{2} h^{\mathrm{T}} h+\frac{1}{\gamma} \dot{\hat{\phi}}\right)-\frac{s^{2}}{2 \delta F}+\frac{\delta}{2} \omega_{0}^{2} \bar{F}_{b}+\frac{\delta}{4} d_{0}^{2}+\frac{\delta}{2} .
\end{aligned}
$$

Considering (25) and (26), we get

$$
\begin{aligned}
\dot{V} & \leq-\kappa \tilde{\phi} \widehat{\phi}-\frac{s^{2}}{2 \delta F}+\frac{\delta}{2} \omega_{0}^{2} \bar{F}_{b}+\frac{\delta}{4} d_{0}^{2}+\frac{\delta}{2} \\
& \leq-\frac{\kappa}{2}\left(\tilde{\phi}^{2}-\phi^{2}\right)-\frac{s^{2}}{2 \delta F}+\frac{\delta}{2} \omega_{0}^{2} \bar{F}_{b}+\frac{\delta}{4} d_{0}^{2}+\frac{\delta}{2} \\
& \leq-\frac{\kappa}{2} \tilde{\phi}^{2}-\frac{s^{2}}{2 \delta F}+\frac{\delta}{2} \omega_{0}^{2} \bar{F}_{b}+\frac{\delta}{4} d_{0}^{2}+\left(\frac{\delta}{2}+\frac{\kappa}{2} \phi^{2}\right) .
\end{aligned}
$$

Setting $\kappa=\eta / \gamma, \eta>0$, it yields

$$
\begin{aligned}
\dot{V} & \leq-\frac{\eta}{2 \gamma} \tilde{\phi}^{2}-\frac{s^{2}}{2 \delta F}+\frac{\delta}{2} \omega_{0}^{2} \bar{F}_{b}+\frac{\delta}{4} d_{0}^{2}+\left(\frac{\delta}{2}+\frac{\eta}{2 \gamma} \phi^{2}\right) \\
& \leq-\alpha V+\beta
\end{aligned}
$$

where $\alpha=\min \{\eta,(1 / \delta)\}, \quad \beta=(\delta / 2) \omega_{0}^{2} \bar{F}_{b}+(\delta / 4) d_{0}^{2}+(\delta / 2)$ $+(\eta / 2 \gamma) \phi^{2}$.

Lemma 1 (see [32]). Let $f, V:[0, \infty) \in R$. Then, $\dot{V} \leq-\alpha_{0}$ $V+f, \forall t \geq t_{0} \geq 0$ implies that

$$
V(t) \leq e^{-\alpha_{0}\left(t-t_{0}\right)} V\left(t_{0}\right)+\int_{t_{0}}^{t} e^{-\alpha_{0}(t-\tau)} f(\tau) d \tau .
$$

Solving the inequality (33) with Lemma 1, we obtain

$$
\begin{aligned}
V(t) & \leq \mathrm{e}^{-\alpha t} V(0)+\beta \times \int_{0}^{t} \mathrm{e}^{-\alpha(t-\tau)} \mathrm{d} \tau \\
& \leq \mathrm{e}^{-\alpha t}\left[V(0)-\frac{\beta}{\alpha}\right]+\frac{\beta}{\alpha}, \quad \forall t \geq 0 .
\end{aligned}
$$

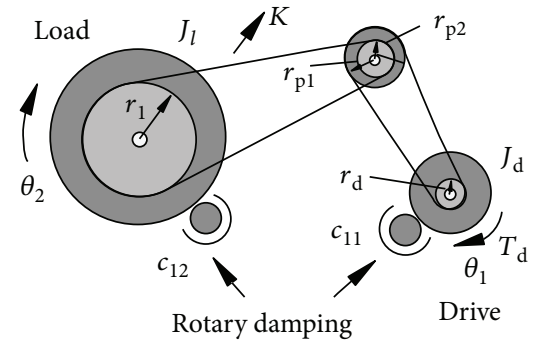

Figure 1: Schematic of the CMD system.

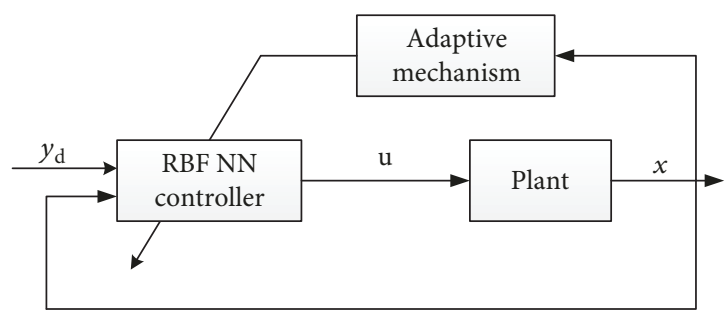

Figure 2: The proposed control scheme.

From the definition of $V$, we obtain

$$
|s| \leq \sqrt{2 F V} \leq \sqrt{2 \bar{F}_{b} V}
$$

According to (30), and $\sqrt{a+b} \leq \sqrt{a}+\sqrt{b}(a>0, b>0)$, we have

$$
|s| \leq \sqrt{2 \bar{F}_{b}}\left(\mathrm{e}^{-\alpha t / 2} \sqrt{V(0)}+\sqrt{\frac{\beta}{\alpha}}\right), \quad \forall t \geq 0
$$

From (37), we conclude that the filtered error function $s$ is bounded such that $\lim _{t \rightarrow \infty}|s| \leq \sqrt{2 \bar{F}_{b} \beta / \alpha}, \forall t \geq 0$, i.e., bounded in the set $S=\left\{s \in R:|s| \leq \sqrt{2 \bar{F}_{b} \beta / \alpha}\right\}$. According to (37), the upper bound of $|s|$ can be arbitrarily small which depends on the appropriate parameters $\alpha$ and $\beta$. $\alpha$ and $\beta$ are related to the parameters $\delta, \eta$, and $\gamma$. Obviously, the increases in $\gamma$ and $\eta$ or decrease in $\delta$ will bring down the upper bound. Moreover, from the definitions of (2), (3), (5), and (10), we conclude that unconstrained tracking error $\xi$ is bounded, indicating the tracking error $e(t)$ is uniformly ultimately bounded and constrained in the compact subset $\mathrm{E}=\left\{e \in R:|e(t)|<\mu_{\infty}\right\}$ for $t \rightarrow \infty$. Hence, the two control objectives that $y$ tracks the desired trajectory $y_{\mathrm{d}}$ and the tracking error $e=y-y_{\mathrm{d}}$ acts as our required performance are achieved. Furthermore, the suitable choice of $\mu(t)$ and $\sigma$ leads to a favorable performance of the system output.

\section{Simulation Results}

In this section, a CMD system is applied to demonstrate the effectiveness of the proposed technique. The schematic of 


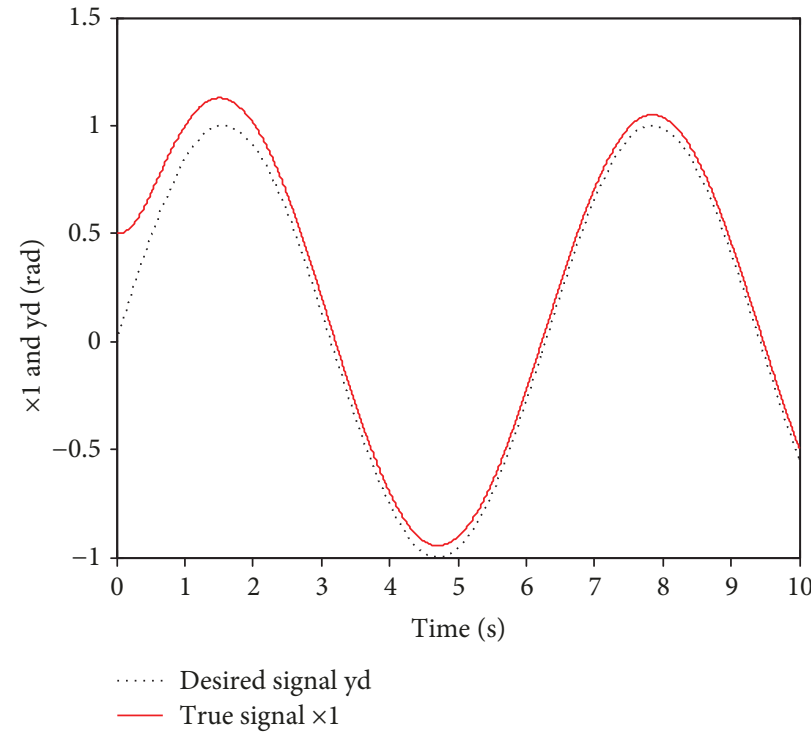

(a)

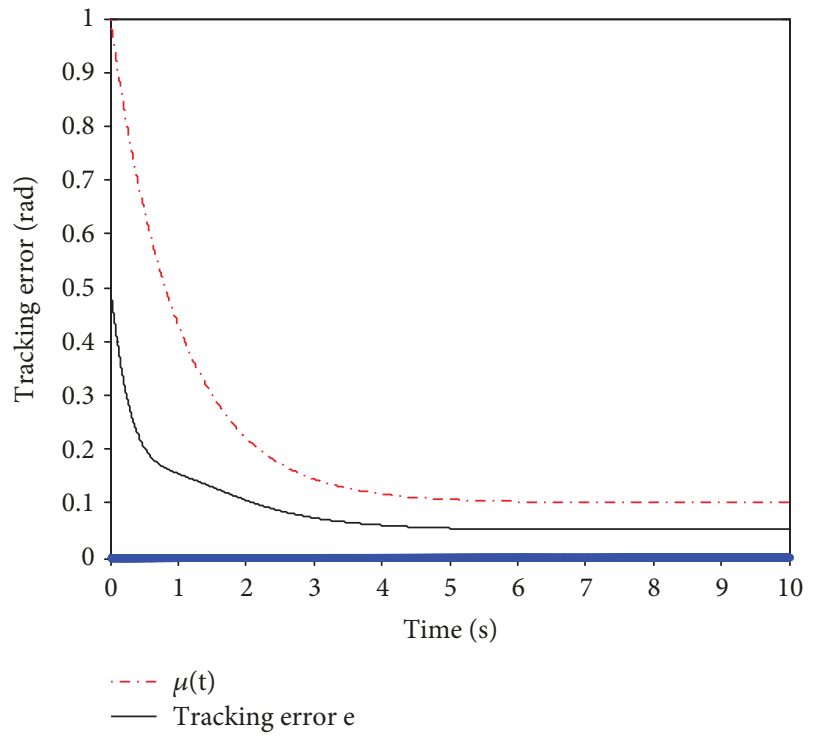

(b)

Figure 3: Position tracking and its tracking error of the closed-loop system with the parameters $l=1, \mu_{\infty}=0.1, \delta=0.5, \gamma=10$, and $\eta=5$ : (a) load angle position $x_{1}$ and desired trajectory $y_{\mathrm{d}}$; (b) tracking error $e=x_{1}-y_{\mathrm{d}}$.

the CMD system is displayed in Figure 1, and the dynamics is given by

$$
\begin{aligned}
J_{1} \ddot{\theta}_{2}+c_{12} \dot{\theta}_{2}+k\left(\theta_{2}-g_{\mathrm{r}}^{-1} \theta_{1}\right) & =0, \\
J_{\mathrm{d}} \ddot{\theta}_{1}+c_{11} \dot{\theta}_{1}+k g_{\mathrm{r}}^{-1}\left(g_{\mathrm{r}}^{-1} \theta_{1}-\theta_{2}\right) & =T_{\mathrm{d}}+d^{\prime}(t),
\end{aligned}
$$

where $J_{\mathrm{d}}$ and $J_{1}$ denote the inertias of drive and load system, respectively; $\theta_{1}$ and $\theta_{2}$ represent the drive angle position and load angle position, respectively; $c_{11}$ and $c_{12}$ are the rotary damping on drive and load; $T_{\mathrm{d}}$ denotes the input torque; $d^{\prime}(t)$ is the unknown disturbance on drive; $g_{\mathrm{r}}=\left(r_{1} r_{\mathrm{pl}}\right) /\left(r_{\mathrm{p} 2} r_{\mathrm{d}}\right)$ is the gear ratio; and $k=2 k_{1} r_{1}^{2}$ is the torsional spring constant.

We set $x_{1}=\theta_{2}$ as the system output, and $u=T_{\mathrm{d}}$ the input, then we could rewrite the dynamics (38) as follows:

$$
\begin{aligned}
J_{1} \ddot{x}_{1}+c_{12} \dot{x}_{1}+k x_{1} & =k g_{\mathrm{r}}^{-1} \theta_{1}, \\
J_{\mathrm{d}} \ddot{\theta}_{1}+c_{11} \dot{\theta}_{1}+k g_{\mathrm{r}}^{-1}\left(g_{\mathrm{r}}^{-1} \theta_{1}-x_{1}\right) & =T_{\mathrm{d}}+d^{\prime}(t) .
\end{aligned}
$$

After elimination of the term $\theta_{1}$ in (39), and setting the time derivatives $\dot{x}_{1}=x_{2}, \dot{x}_{2}=x_{3}$, and $\dot{x}_{3}=x_{4}$, we could obtain the following form as system (1):

$$
\begin{aligned}
\dot{x}_{i} & =x_{i+1}, \quad i=1,2, \ldots, n-1, \\
\dot{x}_{n} & =f(x)+b(x) u+d(t), \\
y & =x_{1},
\end{aligned}
$$

where $n=4, f(x)=-\left(\left(c_{11} k / J_{1} J_{\mathrm{d}}\right)+\left(c_{12} k / J_{1} J_{\mathrm{d}}\right)\left(g_{\mathrm{r}}^{-2}\right)\right)\left(x_{2}\right)-$ $\left(\left(k / J_{1}\right)+\left(c_{11} c_{12} / J_{1} J_{\mathrm{d}}\right)+\left(k / J_{1}\right)\left(g_{\mathrm{r}}^{-2}\right)\right)\left(x_{3}\right)-\left(\left(c_{12} / J_{1}\right)+\left(c_{11} / J_{\mathrm{d}}\right)\right)$ $\left(x_{4}\right), b(x)=k / J_{1} J_{\mathrm{d}} g_{\mathrm{r}}$, and $d(t)=\left(k / J_{1} J_{\mathrm{d}} g_{\mathrm{r}}\right)\left(d^{\prime}(t)\right)$.
As $n=4$, and from (10), we could select the parameters in (10) as $c_{1}=27, c_{2}=27$, and $c_{3}=9$. The initial states of system and the adaptive law (25) are given by $x(0)=$ $\left[\begin{array}{llll}0.5 & 0 & 0 & 0\end{array}\right]^{\mathrm{T}}$ and $\widehat{\phi}(0)=0$. In simulation, the parameter values of system (38) are chosen as $J_{1}=0.3575 \mathrm{~kg} \bullet \mathrm{m}^{2}$, $J_{\mathrm{d}}=0.000425 \mathrm{~kg} \bullet \mathrm{m}^{2}, \quad g_{\mathrm{r}}=4, \quad k=8.45 \mathrm{~N} \cdot \mathrm{m} / \mathrm{rad}, \quad c_{12}=$ $0.004 \mathrm{~N} \bullet \mathrm{m} \bullet \mathrm{s} / \mathrm{rad}, c_{11}=0.05 \mathrm{~N} \bullet \mathrm{m} \bullet \mathrm{s} / \mathrm{rad}$, and the external disturbance $d^{\prime}(t)=0.5 \sin (t)$. The desired trajectory is given by $y_{\mathrm{d}}=\sin t$.

In simulation, a three-layer network 6-9-1 is applied. We choose the input vector as $\psi=\left[x_{1} x_{2} x_{3} x_{4} v s\right]^{\mathrm{T}}$. In order to guarantee RBF vector $h$ be effective, we should make the inputs of RBF be within the effective mapping of Gaussian membership function, which indicates that the center vector $a$ and width value $b_{j}$ should be designed within the effective range of the inputs. In this practical system, we could choose $a=10 \times\left[\begin{array}{lllllllll}-2 & -1.5 & -1 & -0.5 & 0 & 0.5 & 1 & 1.5 & 2\end{array}\right]$ and $b=10$. The proposed scheme is exhibited in Figure 2.

The simulation results are displayed in Figures 3-5. We choose different parameters to illustrate the performance of proposed controller. In Figure 3, the parameters in the controller (21) and adaptive law (25) are chosen as $\delta=0.5, \gamma=10$, and $\eta=5$. The prescribed steady state of the tracking error is given by $\mu_{\infty}=0.1$, the parameter of convergence rate $l=1$ and the overshoot zero such that $\sigma=0$. It is observed that in Figure 3, the tracking error is constrained by the smooth function $\mu(t)$ and along with it. In Figure 4, we increase the convergence rate such that $l=1.5$, and decrease the steady state of the tracking error such that $\mu_{\infty}=0.01$, although the parameters $\delta, \gamma$, and $\eta$ are the same as the values in Figure 3. Then, we further change the parameters in Figure 5 as $\delta=0.05, \gamma=100$, and $\eta=50$, but not regulate $\mu_{\infty}$ and $l$. It can be seen that 


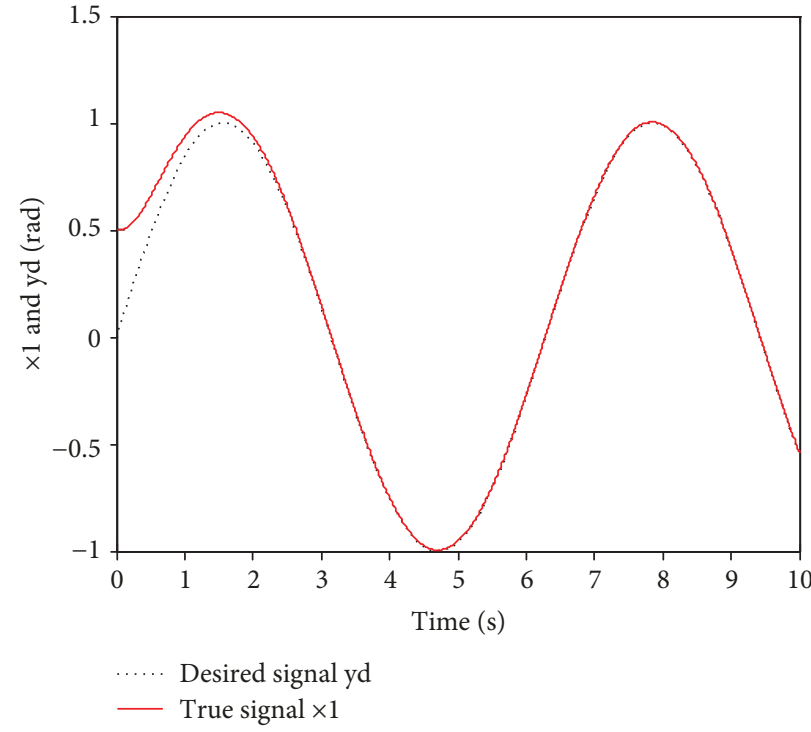

(a)

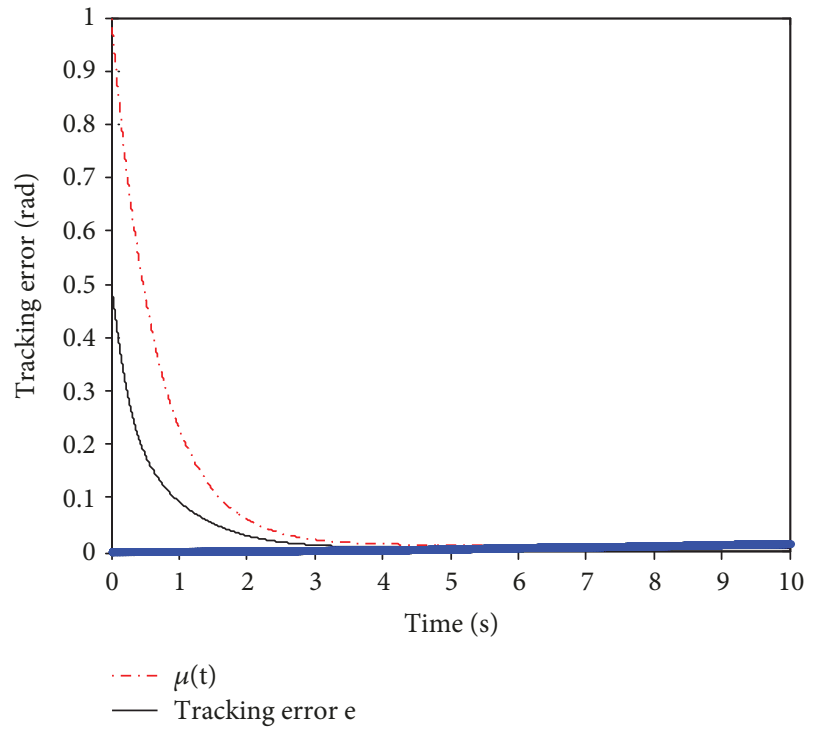

(b)

FiguRe 4: Position tracking and its tracking error of the closed-loop system with the parameters $l=1.5, \mu_{\infty}=0.01, \delta=0.5, \gamma=10$, and $\eta=5$ : (a) load angle position $x_{1}$ and desired trajectory $y_{\mathrm{d}}$; (b) tracking error $e=x_{1}-y_{\mathrm{d}}$.

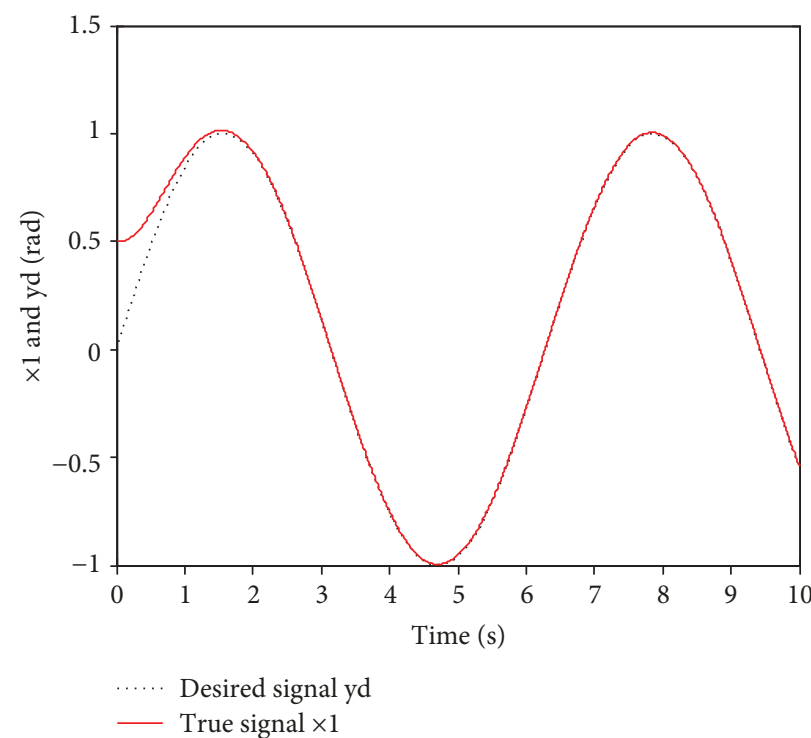

(a)

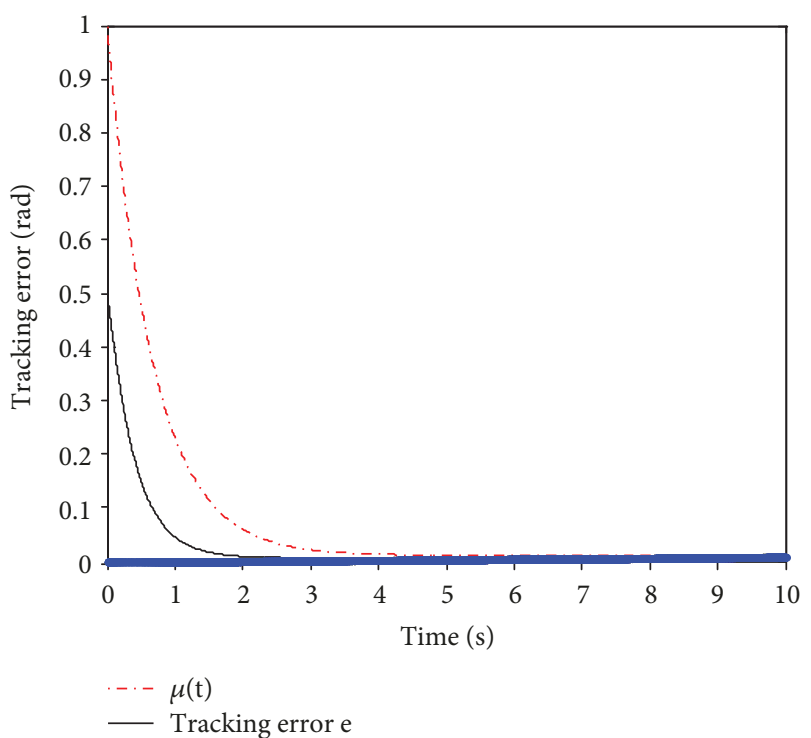

(b)

FIGURE 5: Position tracking and its tracking error of the closed-loop system with the parameters $l=1.5, \mu_{\infty}=0.01, \delta=0.05, \gamma=100$, and $\eta=50$ : (a) load angle position $x_{1}$ and desired trajectory $y_{\mathrm{d}}$; (b) tracking error $e=x_{1}-y_{\mathrm{d}}$.

Figures 3-5 illustrate the different performances of tracking error. From the comparison, we validate that the smooth function $\mu(t)$ can effectively constrain the tracking error to act as our required performance. The suitable choice of $\mu(t)$ and $\sigma$ leads to a favorable performance of output. Meanwhile, the smaller $\delta$ and larger $\gamma$ and $\eta$ will accelerate the convergence rate of the closed-loop system and the tracking error could be arbitrarily small. Simulations illustrate the effectiveness of the proposed scheme.

\section{Conclusion}

A tracking-error-constrained controller is proposed in this study for a class of unknown nonlinear systems with external disturbances. We develop the controller to achieve that the system tracking error acts as our required performance. The ideal controller with unknown smooth functions is designed to obtain the actual one using a single parameter RBF NN adaptive control method. The developed adaptive 
control law guarantees the stability of the closed-loop system via the theoretical analysis. The simulations illustrate a favorable tracking performance, and the tracking error could be arbitrarily small by selecting the appropriate parameters.

\section{Data Availability}

The data used to support the findings of this study are available from the corresponding author upon request.

\section{Conflicts of Interest}

The authors declare that they have no conflicts of interest.

\section{Acknowledgments}

This work was partially supported by the National Natural Science Foundation of China (Grant no. 61703402).

\section{References}

[1] C. Yang, Y. Jiang, Z. Li, W. He, and C.-Y. Su, "Neural control of bimanual robots with guaranteed global stability and motion precision," IEEE Transactions on Industrial Informatics, vol. 13, no. 3, pp. 1162-1171, 2017.

[2] C. Yang, X. Wang, L. Cheng, and H. Ma, "Neural-learningbased telerobot control with guaranteed performance," IEEE Transactions on Cybernetics, vol. 47, no. 10, pp. 3148-3159, 2017.

[3] W. He, T. Meng, X. He, and C. Sun, "Iterative learning control for a flapping wing micro aerial vehicle under distributed disturbances," IEEE Transactions on Cybernetics, pp. 1-12, 2018.

[4] P. Moallem, N. Razmjooy, and M. Ashourian, "Computer vision-based potato defect detection using neural networks and support vector machine," International Journal of Robotics and Automation, vol. 28, no. 2, pp. 137-145, 2013.

[5] C. Yang, J. Luo, Y. Pan, Z. Liu, and C.-Y. Su, "Personalized variable gain control with tremor attenuation for robot teleoperation," IEEE Transactions on Systems, Man, and Cybernetics: Systems, pp. 1-12, 2017.

[6] W. He, Z. Yan, C. Sun, and Y. Chen, "Adaptive neural network control of a flapping wing micro aerial vehicle with disturbance observer," IEEE Transactions on Cybernetics, vol. 47, no. 10, pp. 3452-3465, 2017.

[7] C. Yang, Y. Jiang, W. He, J. Na, Z. Li, and B. Xu, “Adaptive parameter estimation and control design for robot manipulators with finite-time convergence," IEEE Transactions on Industrial Electronics, vol. 65, no. 10, pp. 8112-8123, 2018.

[8] S. Zhang, Y. Dong, Y. Ouyang, Z. Yin, and K. Peng, “Adaptive neural control for robotic manipulators with output constraints and uncertainties," IEEE Transactions on Neural Networks and Learning Systems, pp. 1-11, 2018.

[9] W. He and Y. Dong, "Adaptive fuzzy neural network control for a constrained robot using impedance learning," IEEE Transactions on Neural Networks and Learning Systems, vol. 29, no. 4, pp. 1174-1186, 2018.

[10] C. Yang, H. Wu, Z. Li, W. He, N. Wang, and C.-Y. Su, "Mind control of a robotic arm with visual fusion technology," IEEE Transactions on Industrial Informatics, p. 1, 2017.

[11] J. Fan, S. Jia, and X. Li, "Direct adaptive control based on improved RBF neural network for omni-directional mobile robot," in Proceedings of the 2015 International Conference on Mechatronics, Electronic, Industrial and Control Engineering, Shenyang, China, 2015, Atlantis Press.

[12] Y. Fang, J. Fei, and K. Ma, "Model reference adaptive sliding mode control using RBF neural network for active power filter," International Journal of Electrical Power \& Energy Systems, vol. 73, pp. 249-258, 2015.

[13] B. Huo, Y. Xia, S. Chai, and P. Shi, "Adaptive fault-tolerant control of rigid body using RBF neural networks," in Proceeding of the 11th World Congress on Intelligent Control and Automation, pp. 1185-1190, Shenyang, China, 2014, IEEE.

[14] W. He and S. S. Ge, "Cooperative control of a nonuniform gantry crane with constrained tension," Automatica, vol. 66, no. 4, pp. 146-154, 2016.

[15] B. Niu and J. Zhao, "Tracking control for output-constrained nonlinear switched systems with a barrier Lyapunov function," International Journal of Systems Science, vol. 44, no. 5, pp. 978-985, 2013.

[16] K. P. Tee, S. S. Ge, and E. H. Tay, "Barrier Lyapunov functions for the control of output-constrained nonlinear systems," Automatica, vol. 45, no. 4, pp. 918-927, 2009.

[17] B. Ren, S. S. Ge, K. P. Tee, and T. H. Lee, "Adaptive neural control for output feedback nonlinear systems using a barrier Lyapunov function," IEEE Transactions on Neural Networks, vol. 21, no. 8, pp. 1339-1345, 2010.

[18] W. He, X. He, M. Zou, and H. Li, "PDE model-based boundary control design for a flexible robotic manipulator with input backlash," IEEE Transactions on Control Systems Technology, pp. 1-8, 2018.

[19] Y. Karayiannidis and Z. Doulgeri, "Model-free robot joint position regulation and tracking with prescribed performance guarantees," Robotics and Autonomous Systems, vol. 60, no. 2, pp. 214-226, 2012.

[20] A. K. Kostarigka, Z. Doulgeri, and G. A. Rovithakis, "Prescribed performance tracking for flexible joint robots with unknown dynamics and variable elasticity," Automatica, vol. 49, no. 5, pp. 1137-1147, 2013.

[21] W. He, T. Meng, X. He, and S. S. Ge, "Unified iterative learning control for flexible structures with input constraints," Automatica, vol. 96, pp. 326-336, 2018.

[22] C. P. Bechlioulis and G. A. Rovithakis, "Robust partial-state feedback prescribed performance control of cascade systems with unknown nonlinearities," IEEE Transactions on Automatic Control, vol. 56, no. 9, pp. 2224-2230, 2011.

[23] H. Yang and J. Liu, “An adaptive RBF neural network control method for a class of nonlinear systems," IEEE/CAA Journal of Automatica Sinica, vol. 5, no. 2, pp. 457-462, 2018.

[24] H. J. Yang and M. Tan, "Sliding mode control for flexible-link manipulators based on adaptive neural networks," International Journal of Automation and Computing, vol. 15, no. 2, pp. 239-248, 2018.

[25] H. Yang and Z. Liu, "Active control of an elastic beam based on state and input constraints," IEEE Access, vol. 6, no. 1, pp. 10635-10643, 2018.

[26] H. Yang and J. Liu, “Active vibration control for a flexible-link manipulator with input constraint based on a disturbance observer," Asian Journal of Control, 2018.

[27] S. S. Ge, C. C. Hang, and T. Zhang, "A direct method for robust adaptive nonlinear control with guaranteed transient performance," Systems \& Control Letters, vol. 37, no. 5, pp. 275-284, 1999. 
[28] F. L. Lewis, K. Liu, and A. Yesildirek, "Neural net robot controller with guaranteed tracking performance," IEEE Transactions on Neural Networks, vol. 6, no. 3, pp. 703-715, 1995.

[29] O. Kuljaca, N. Swamy, F. L. Lewis, and C. M. Kwan, “Design and implementation of industrial neural network controller using backstepping," IEEE Transactions on Industrial Electronics, vol. 50, no. 1, pp. 193-201, 2003.

[30] W. He, Z. Li, and C. L. P. Chen, "A survey of human-centered intelligent robots: issues and challenges," IEEE/CAA Journal of Automatica Sinica, vol. 4, no. 4, pp. 602-609, 2017.

[31] H. K. Khalil and J. Grizzle, Nonlinear Systems, vol. 3, Prentice Hall, Upper Saddle River, NJ, USA, 2002.

[32] P. A. Ioannou and J. Sun, Robust Adaptive Control, Dover Publications, 2012. 


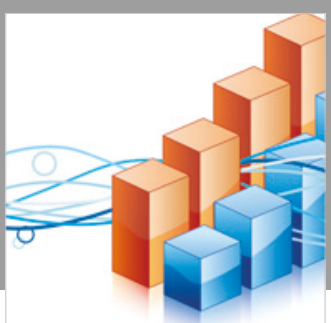

Advances in

Operations Research

\section{-n-m}
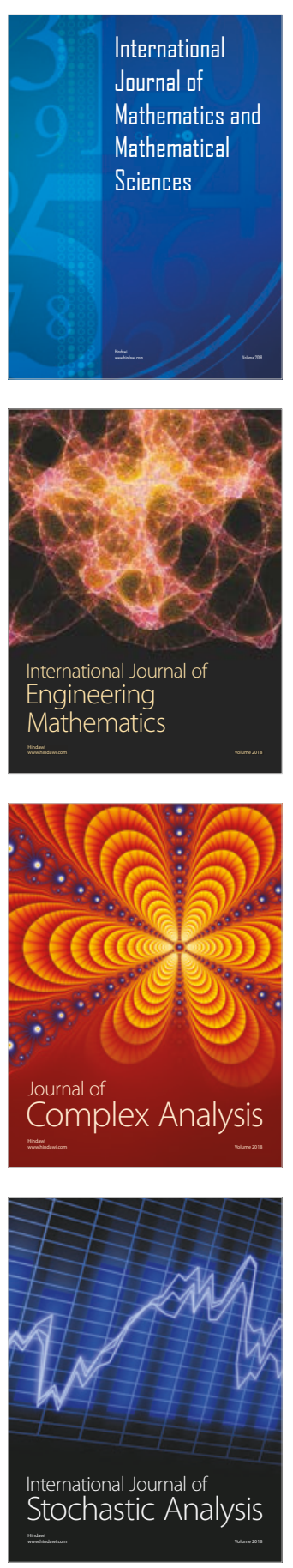
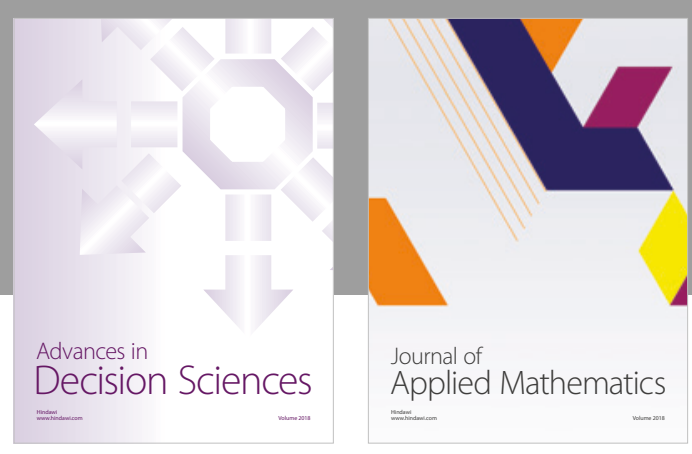

Journal of

Applied Mathematics
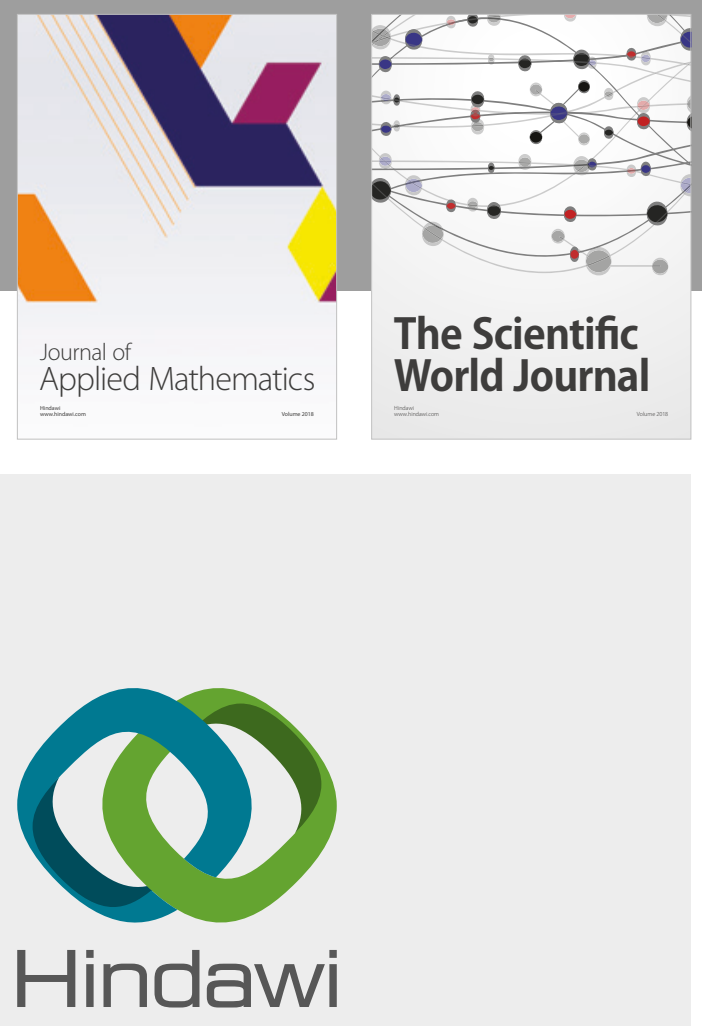

Submit your manuscripts at

www.hindawi.com

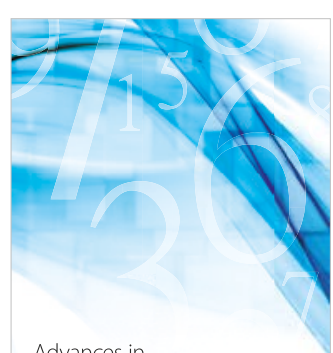

Advances in
Numerical Analysis
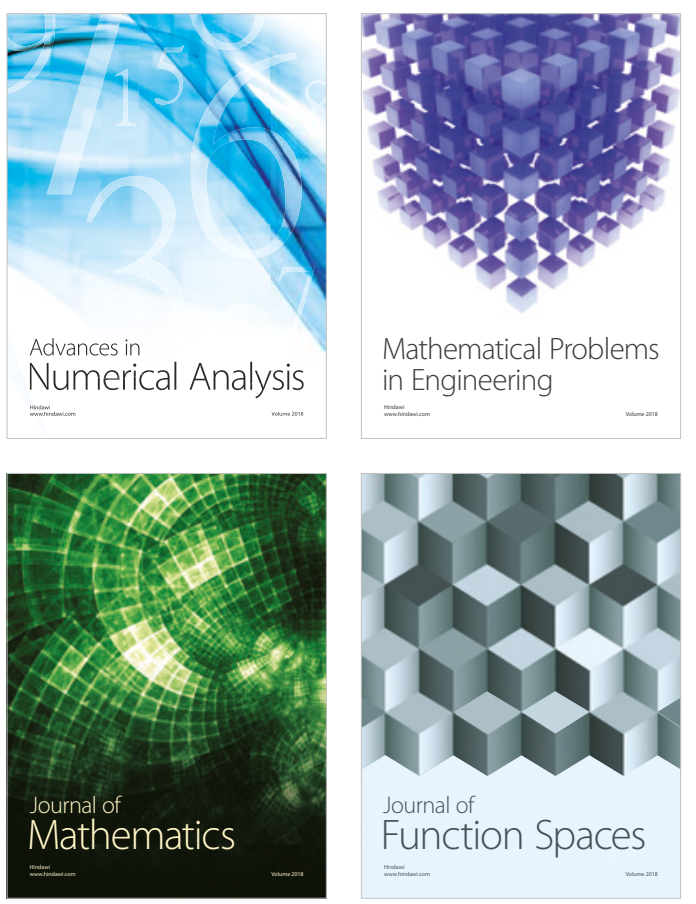

Mathematical Problems in Engineering

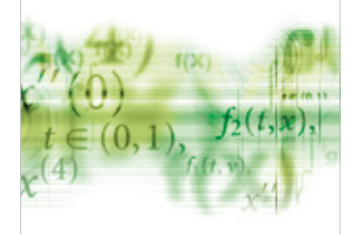

International Journal of

Differential Equations

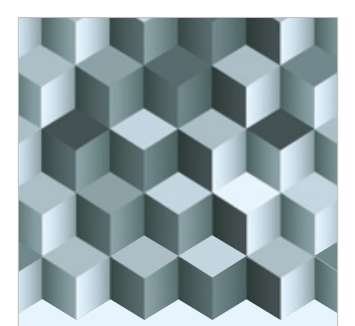

Journal of

Function Spaces
The Scientific

World Journal

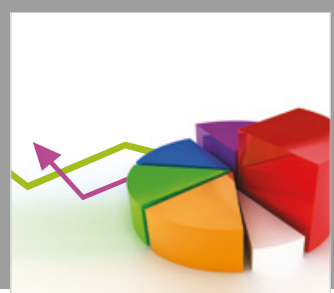

Journal of

Probability and Statistics
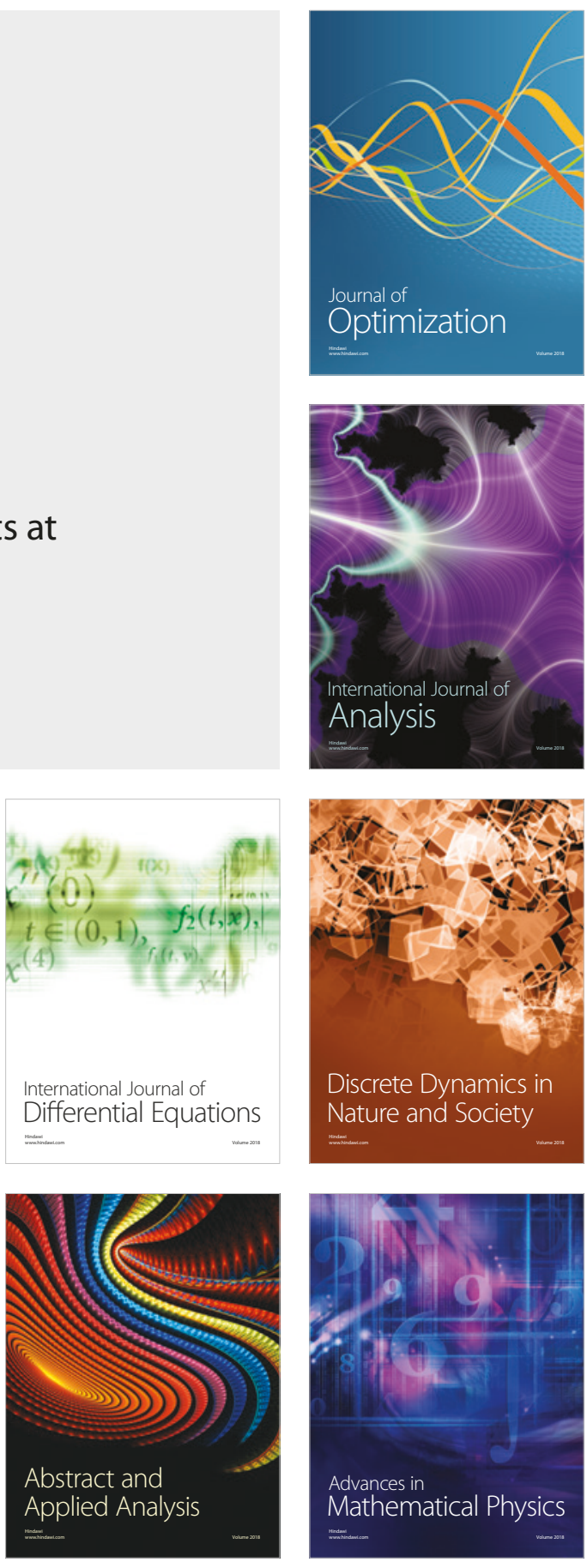